\title{
The tightrope of science, media and politics
}

\author{
The urgent need to address COVID-19 has highlighted the delicate relationships among science, politics and the \\ media. To achieve a successful long-term response to the pandemic, stakeholders need to be guided by data, \\ integrity and a sense of responsibility toward the public.
}

T here are, indeed, two things, knowledge and opinion, of which the one makes its possessor really to know, the other to be ignorant," says Hippocrates in his Law. These words resonate profoundly in the midst of the COVID-19 pandemic, when the urgent pursuit of scientific knowledge on the causative virus SARS-CoV-2 contrasts so starkly with a cacophony of uninformed opinions on the same topic.

On the positive side, the world has witnessed an extraordinary shift in the way research is conducted and results are publicized. The pressing need to address this public-health emergency has led to a sharp refocusing of research efforts on COVID-19 and pooling of expertise and resources. These efforts include the establishment of international collaborative networks among researchers and clinicians in both academia and industry, alongside community-led initiatives to gather large-scale clinical and epidemiological information ${ }^{1}$. Science has traditionally been an international endeavor. However, the scale and single focus of this global scientific mobilization is unprecedented. Scientists have taken advantage of all the means technology and publishing afford to increase communication and dissemination of information in near real time across the globe. Preprint servers are being widely used as the first port of data-sharing with the community. Publishers, including Springer Nature, have been expediting the peer review of COVID-19 studies and have been working with global organizations, including the World Health Organization, to ensure that all relevant research is immediately available. Social-media platforms have become hotbeds of scientific discourse and critique of the latest COVID-19 developments. As a result, our understanding of viral biology and infection, disease presentation and epidemiology has increased rapidly, which has led to the swift development of tests, preclinical and clinical evaluation of numerous treatment approaches and vaccines, and data-driven guidance for disease treatment and containment.

The deluge of COVID-19-related information, coupled with the urgent need for reliable medical solutions and keeping the public informed, has accentuated crucial pitfalls in science communication. For instance, although preprints are an established method of sharing non-peer-reviewed, more-preliminary findings, their reporting has not always escaped sensationalism. A prominent example is a - now-withdrawn - preprint ${ }^{2}$ that was interpreted as support for the virus having been engineered, which fueled conspiracy theories about its origins, some of which were unfortunately repeated by certain government officials. More recently, a preprint extrapolating on the transmissibility of SARS-CoV-2 based solely on computational analyses of mutations in a single viral protein ${ }^{3}$ was reported as evidence of a new, dominant and more-contagious strain of the virus. The scientific community was quick to critique the findings; however, the risks of inaccurate reporting of preliminary results cannot be overstated. To mitigate these risks, servers such as bioRxiv and medRxiv include prominent disclaimers warning that preprints "should not be regarded as conclusive, guide clinical practice/health-related behavior, or be reported in news media as established information." Researchers are also helping to evaluate and publicize high-quality preprints, as exemplified by the joint effort to assess immunology-focused COVID-19 preprints by scientists at the Icahn School of Medicine at Mount Sinai, New York, and our sister title Nature Reviews Immunology.

On the therapy front, long-awaited good news came in the form of the preliminary but encouraging results of a clinical trial sponsored by the US National Institute of Allergy and Infectious Diseases that led to the emergency use authorization of remdesivir as a potential COVID-19 treatment by the US Food and Drug Administration (FDA). This approval comes on the heels of positive results announced from a separate trial run by the drug's manufacturer, which, however, appears to lack a no-drug control arm and thus should be interpreted very cautiously. More sobering are the findings of a third clinical trial from China that reported no benefit for patients compared with placebo controls ${ }^{4}$ and underscored the fact that more work is needed to verify whether remdesivir is in fact beneficial for patients.

Obtaining conflicting results is of course not surprising to researchers and clinicians. Scientific progress occurs through trial, error and correction based on careful evaluation of the available data and their limitations. A large part of the media, especially those with dedicated science and medicine reporters, routinely provide informed coverage with these principles in mind and have been doing so diligently during the pandemic. However, hype, false hope and harm from erroneously touted COVID-19 therapies have not been avoided. Hydroxychloroquine and chloroquine have quickly become a cautionary tale against promoting potential benefits of a drug prematurely. Propelled to the limelight of the 24-hour COVID-19 news cycle on the basis of early indications of in vitro efficacy against SARS-CoV- $2^{5}$ and a subsequent emergency use authorization by the FDA, these drugs were touted widely by sections of the media and very prominent politicians as a game-changer in the fight against COVID-19. Despite scientists' warnings that conclusive results were not yet available, the persistent hype resulted in panic-buying, drug shortages and attempts to self-medicate ${ }^{6}$ with tragic consequences. The hype died down only following reports of serious adverse events, including potential death, that led the FDA to caution against use outside a hospital or clinical trial setting.

Science and politics have always been in a close but uneasy relationship. In an ideal world, politics (and, by extension, the society that shapes it) would support the reasonable scope of science without serving secondary political interests; strong science would inform politics and policy decisions; while a free and responsible press would hold both to account. Ours not being an ideal world, science and politics are often at cross purposes, with some politicians discounting scientific evidence that may not support their political needs, and science striving to maintain independence from political will, while requiring its support for infrastructure and funding.

Some of these conflicts have become apparent during the response of different countries to the pandemic. 
Several European and Asia-Pacific countries made science-driven decisions to stem the spread of infection, established preventative measures, such as social distancing and use of face masks by the general public, and implemented widespread testing and contact tracing. Some of these countries are now reporting results that point to success in containing the virus ${ }^{7}$ and are moving toward easing restrictions and reopening their economies. On the flipside, the USA and UK have the unenviable position of being the epicenters of the pandemic globally and in Europe, respectively. The fact that they have been hit so hard is due in no small part to their governments' not heeding scientific recommendations to contain infections and fortify their healthcare systems. As a result, they mounted delayed and poorly planned responses that in some cases included conflicting guidance to the public. Despite the continued lack of concrete systems to enable the identification and isolation of new cases to prevent future infection spikes, the political discourse, including by certain parts of the media, has shifted to reopening the economies of these countries as well. The arguments for this are undeniably strong. Extended lockdown is unsustainable for individual health and wellbeing, for people's livelihoods and also for the broader economy. Indeed, the COVID-19 pandemic has brought on a deep economic recession that will require strong measures and political courage to address. However, in the absence of disease containment and realistic plans to prevent and control future outbreaks, lifting preventative measures prematurely simply demonstrates that we have learned little from the dire experiences of the past few months and thus are likely to repeat them, with the devastating loss of life that would entail.

Where do scientists fit in this particular debate? They are essential to the formation of sound public-health policy, but perhaps for some politicians and members of the press and public they represent the uncomfortable uncertainties that characterize this pandemic. Rather than providing the clear, fast solutions and magic-bullet cures that would allow life to return to normal, scientists offer tentative answers, look for the cracks in the data, signpost caveats and ask for more time. That is typical of the scientific method, but it clashes with the human need for clarity and hope.

Going back to the words of Hippocrates about knowledge versus opinion, it is the responsibility of scientists to help shape public opinion on the basis of reliable knowledge. Scientists today are called to tread the delicate line of building public trust while providing sober assessments of data and managing expectations.
The increased connectivity of our digital times means that it is easier to engage directly and disseminate knowledge in simple, digestible bites. However, maintaining an honest working balance with politics and media is also crucial, given that science does not operate in a vacuum. Rebutting misinformation and pseudoscience quickly and emphatically is essential. This also requires stepping out of our individual professional and social echo chambers, self-affirming and comfortable though they may feel, to engage with the other - mistrusting - side. Now more than ever we need to remember that, similar to pandemic cures, change in public opinion does not come about with miracles, but through dialog, respect and incremental but decisive steps.

Published online: 14 May 2020 https://doi.org/10.1038/s43018-020-0073-z

References

1. Desai, A. et al. Nat. Cancer https://doi.org/10.1038/s43018-0200065-z (2020).

2. Pradhan, P. et al. bioRxiv https://doi.org/10.1101/2020.01.30 927871 (2020).

3. Korber, B. et al. bioRxiv https://doi.org/10.1101/2020.04.29. 069054 (2020).

4. Wang, Y. Lancet https://doi.org/10.1016/S0140-6736(20)31022-9 (2020).

5. Wang, M. et al. Cell Res. 30, 269-271 (2020).

6. Brian, Owens Lancet Rheumatol. 2, e257 (2020).

7. Gibney, E. Nature 581, 15-16 (2020) 